\title{
Alantolactone induces apoptosis in RKO cells through the generation of reactive oxygen species and the mitochondrial pathway
}

\author{
YU ZHANG $^{1,2}$, YONG LI BAO ${ }^{1}$, YIN WU ${ }^{2}$, CHUN LEI YU ${ }^{1,2}$, \\ YAN XIN HUANG ${ }^{3}$, YING SUN ${ }^{3}$, LI HUA ZHENG ${ }^{3}$ and YU XIN $\mathrm{LI}^{2,3}$ \\ ${ }^{1}$ National Engineering Laboratory for Druggable Gene and Protein Screening; \\ ${ }^{2}$ Research Center of Agriculture and Medicine Gene Engineering of Ministry of Education; \\ ${ }^{3}$ Institute of Genetics and Cytology, Northeast Normal University, Changchun, Jilin 130024, P.R. China
}

Received March 2, 2013; Accepted June 19, 2013

DOI: $10.3892 / \mathrm{mmr} .2013 .1640$

\begin{abstract}
Alantolactone, a methanol extract of Inula helenium, possesses anticancer properties in a number of cancer cell lines. However, its anticancer effect on human colorectal cancer cells and the underlying mechanisms remain to be elucidated. In the present study, the effects of alantolactone on cell viability and apoptosis in RKO human colon cancer cells were investigated. Alantolactone treatment of RKO cells was found to result in dose-dependent inhibition of cell viability and induction of apoptosis, accompanied with the accumulation of reactive oxygen species (ROS) and the disruption of mitochondrial membrane potential. In addition, these effects were blocked with $\mathrm{N}$-acetylcysteine, a specific ROS inhibitor. Western blotting indicated that exposure of RKO cells to alantolactone is associated with the downregulation of Bcl-2, induction of Bax and activation of caspase-3 and -9. These results indicated that a ROS-mediated mitochondria-dependent pathway is involved in alantolactone-induced apoptosis. From these observations, it was hypothesized that alantolactone may be used for the treatment of human colon cancer.
\end{abstract}

\section{Introduction}

Colorectal cancer is the most common type of cancer and the leading cause of cancer-related mortality worldwide (1).

Correspondence to: Dr Yong Li Bao, National Engineering Laboratory for Druggable Gene and Protein Screening, Northeast Normal University, 5268 Renmin Street, Changchun, Jilin 130024, P.R. China

E-mail: baoyl800@nenu.edu.cn

Dr Yu Xin Li, Research Center of Agriculture and Medicine Gene Engineering of Ministry of Education, Northeast Normal University, 5268 Renmin Street, Changchun, Jilin 130024, P.R. China

E-mail: liyx486@nenu.edu.cn

Key words: alantolactone, colorectal cancer, nature compounds, apoptosis
There is a continuing need to develop safe and effective anticancer drugs as current surgery and chemotherapeutic options appear to be inadequate in curing or controlling colorectal cancer (2).

Previously, plant-derived drugs have been important in cancer therapy due to their low toxicity and high efficacy. Numerous natural bioactive compositions found in medicinal herbs are considered to be potential anticancer agents (3-5). Alantolactone (Fig. 1), a sesquiterpene lactone, is the active component of Inula helenium (Compositae), a traditional Chinese herbal medicine which has strong anthelmintic and antibacterial activities (6) and possesses inhibitory activities against human gastric adenocarcinoma MK-1 cells, human uterine carcinoma HeLa cells and mouse melanoma B16F10 cells (7). In addition, alantolactone induces apoptosis in Jurkat leukemia T cells (8) and human chronic myelogenous leukemia K562 cells (9). It has previously been observed that alantolactone inhibits HCT-8 cell proliferation (10). However, its antitumor effect on colorectal cancer cells and the underlying mechanisms involved have not been fully characterized.

Apoptosis is a programmed cell death process that regulates normal development and homeostasis in organisms. A contributing factor to the survival of tumor cells is loss of apoptotic control, thus, numerous anticancer agents enhance cancer cell apoptosis to control cancer development $(11,12)$. Apoptosis is characterized by distinct morphological changes, including membrane blebbing, cell shrinkage, loss of mitochondrial membrane potential (MMP), chromatin condensation and DNA fragmentation $(12,13)$. At the biochemical level, apoptosis is mediated by the activation of a class of cysteine proteases known as caspases. In mammalian cells, caspase activation mainly occurs via death receptor activation or mitochondrial membrane depolarization (14). Mitochondrial-dependent apoptosis is regulated principally by the Bcl-2 protein family. In response to apoptotic signals, Bax, a proapoptotic member of the Bcl-2 family, is redistributed from the cytosol to the mitochondria (3). The ratio of expression of Bax protein and Bcl-2 protein ultimately determines cell death or survival in the mitochondrial apoptotic pathway (15). 
Reactive oxygen species (ROS) play a key role in mitochondria-mediated apoptosis. ROS are the byproducts of aerobic respiration and primarily arise from the mitochondria $(16,17)$. At low concentrations, ROS has been identified as a second messenger in signaling pathways. However, high levels of ROS in the mitochondria may cause mitochondrial membrane depolarization, the release of mitochondrial factors and triggering of caspase cascades (18). Under normal conditions, the majority of anticancer drugs target mitochondrial function, producing ROS (19-22). Therefore, ROS is important in mitochondria-mediated apoptosis.

The aim of the present study was to explore the cytotoxic activity of alantolactone on human RKO colon cancer cells and its underlying mechanisms, through an analysis of the ROS-mediated mitochondrial pathway, accumulation of intracellular ROS and the disruption of MMP. In addition, the expression of Bcl-2, Bax and activated caspase-3 and -9 was determined.

\section{Materials and methods}

Cell lines. RKO were cultured in RPMI-1640 medium with $10 \%$ fetal bovine serum (TBD Science, Tianjin, China), $100 \mathrm{U} / \mathrm{ml}$ penicillin and $100 \mu \mathrm{g} / \mathrm{ml}$ streptomycin, at $37^{\circ} \mathrm{C}$ and $5 \% \mathrm{CO}_{2}$.

Antibodies and reagents. Antibodies against $\mathrm{Bax}$ and $\mathrm{Bcl}-2$ were purchased from Santa Cruz Biotechnology, Inc. (Santa Cruz, CA, USA) and antibodies against cleaved caspase-3 and -9 were purchased from Cell Signaling Technology Inc., (Beverly, MA, USA). A mouse monoclonal antibody against GAPDH was purchased from KangCheng Bio-tech (Shanghai, China). DAPI was purchased from the Beyotime Institute of Biotechnology (Shanghai, China). DCFH-DA, $\mathrm{N}$-acetylcysteine (NAC) and $\operatorname{DiOC}_{6}(3)$ were purchased from Sigma-Aldrich (St. Louis, MO, USA).

Cell proliferation analysis. Effect of alantolactone on RKO cell viability was determined by MTT assay. Cells ( $1 \times 10^{4}$ cells/well) were plated in 96-well plates for $24 \mathrm{~h}$ and treated with $1.25,2.5,5$ and $10 \mu \mathrm{g} / \mathrm{ml}$ alantolactone in the presence of 3\% serum. Following 1,2,3 and 4 day treatment, $20 \mu 1$ MTT(5 mg/ml; Sigma-Aldrich) was added to each well for an additional 4-h incubation. The experiment was performed as described previously (23).

Nuclei staining. RKO cells were treated with $0,2.5$ and $5 \mu \mathrm{g} / \mathrm{ml}$ alantolactone. The experiment was performed as described previously (24). Analyses were performed in triplicate and a minimum of 100 cells/field and at least four fields in each well were counted.

TUNEL assay. TUNEL assay was performed using an Apoptosis Detection kit (Roche Diagnostics GmbH, Steinheim, Germany). Cells were treated with $2.5 \mu \mathrm{g} / \mathrm{ml}$ alantolactone for $24 \mathrm{~h}$ prior to being harvested. The cells were then washed with PBS, fixed with $3.7 \%$ paraformaldehyde for $10 \mathrm{~min}$ at room temperature and stained with a TUNEL solution for $30 \mathrm{~min}$ at $37^{\circ} \mathrm{C}$, followed by DAPI staining. The cells were imaged using an inverted fluorescence microscopy (Olympus BX50, Tokyo, Japan).
Protein extraction and western blotting. Following alantolactone treatment for $24 \mathrm{~h}$, the cells were harvested and treated with lysis buffer (Beyotime Institute of Biotechnology). Western blotting was performed as described previously (24).

Plasmid transfection. Subcellular localization vector was used to label mitochondria (mito-GFP, signal peptide sequence of cytochrome $c$ oxidase VII (25), was inserted upstream of GFP between the HindIII and EcoRI restriction sites in the pcDNA3 vector). The plasmids were transfected using Lipofectamine ${ }^{\mathrm{TM}}$ 2000 (Invitrogen Life Technologies, Carlsbad, CA, USA) according to the manufacturer's instructions.

Detection of ROS levels. RKO cells were treated with $2.5 \mu \mathrm{g} / \mathrm{ml}$ alantolactone for $24 \mathrm{~h}$. Cells were washed twice with PBS and incubated with DCFH-DA (final concentration, $40 \mu \mathrm{M}$ ) for $30 \mathrm{~min}$ in the dark at $37^{\circ} \mathrm{C}$. Fluorescent images were immediately captured under an inverted fluorescence microscope (Olympus BX50).

Determination of MMP. RKO cells were treated with alantolactone for $12 \mathrm{~h}$, washed and incubated with $\mathrm{DiOC}_{6}(3)(50 \mathrm{nM}$ final) in PBS for $30 \mathrm{~min}$ in the dark at $37^{\circ} \mathrm{C}$. The cells were washed with PBS twice and the fluorescence was immediately measured with FLUOstar OPTIMA (BMG LABTECH GmbH, Ortenberg, Germany) using $507 \mathrm{~nm}$ EX and 529 nm EM filter settings.

Statistical analysis. All the presented data and results were confirmed in at least three independent experiments. Statistical comparisons were made using Student's t-test. ${ }^{*} \mathrm{P}<0.05$ and ${ }^{* *} \mathrm{P}<0.01$ were considered to indicate statistically significant differences. Error bars denote the standard deviation (SD).

\section{Results}

Alantolactone inhibits cell proliferation in RKO cells. An MTT assay was used to determine the effect of alantolactone on cell viability under various concentrations of alantolactone $(1.25,2.5,5$ and $10 \mu \mathrm{g} / \mathrm{ml})$ and following 1, 2, 3 and 4 days of treatment. Alantolactone was found to inhibit RKO cell viability in a dose- and time-dependent manner (Fig. 2A). Thus, it was hypothesized that alantolactone is capable of markedly inhibiting colon cancer proliferation.

Alantolactone induces apoptosis in RKO cells. To test whether alantolactone-mediated growth inhibition was due to induction of apoptosis, DAPI staining and TUNEL assay were performed to analyze the effects of alantolactone on apoptosis in RKO cells. DAPI staining revealed that the control cells were round with homogeneous nuclei, by contrast, alantolactone-treated cells showed condensed and fragmented nuclei (arrows; Fig. 3A). In addition, alantolactone was observed to induce apoptosis in a dose-and time-dependent manner (Fig. 3B). Results obtained from TUNEL assay (Fig. 3C) suggested that alantolactone may significantly induce apoptosis in RKO cells.

Alantolactone causes the generation of ROS. As ROS generation is important in apoptosis, the effect of alantolactone on 


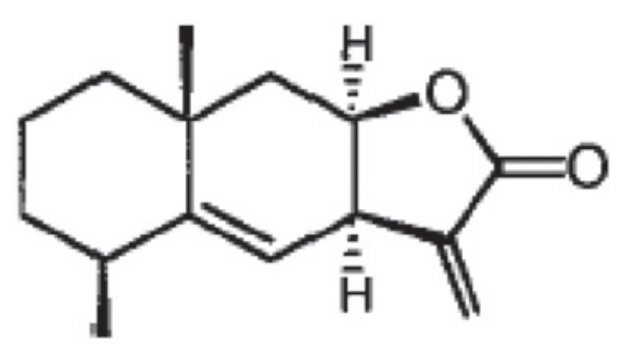

Figure 1. Chemical structure of alantolactone.

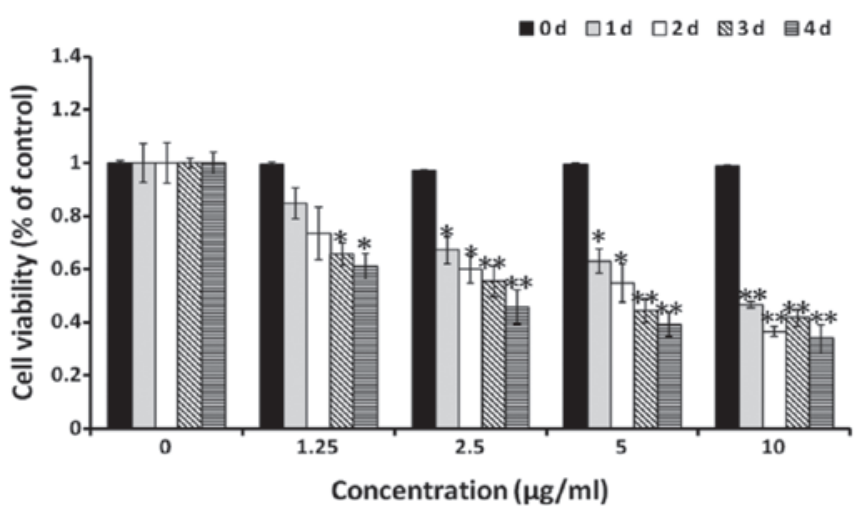

Figure 2. Effects of alantolactone on cell growth in colon cancer cells. Cells were treated with vehicle or various concentrations of alantolactone for 4 days. Data are presented as the relative proportion of viable cells (\%) by comparing the alantolactone-treated group with the vehicle-treated cells, the viability of which was assumed to be $100 \%$. The results are presented as the mean $\pm \mathrm{SD}$ of three independent experiments. ${ }^{*} \mathrm{P}<0.05$ and ${ }^{* * *} \mathrm{P}<0.01$, vs. vehicle-treated cells.

the generation of ROS was investigated. Cells were exposed to alantolactone $(2.5 \mu \mathrm{g} / \mathrm{ml})$ for $24 \mathrm{~h}$ and analyzed for the accumulation of ROS by fluorescence microscopy following staining with DCFH-DA. As shown in Fig. 4A, treatment with alantolactone resulted in a significant increase in intracellular ROS in RKO cells. NAC is a potent antioxidant that may inhibit oxidative stress by directly scavenging ROS and replenishing GSH (26). The decrease of intracellular ROS by NAC was also observed following DCFH-DA staining (Fig. 4A).

To investigate the role of ROS in alantolactone-induced apoptosis in RKO cells, cell death was measured following treatment of alantolactone only or with NAC. As shown in Fig. 4B, alantolactone $(2.5 \mu \mathrm{g} / \mathrm{ml})$ increased cell death, whereas removing intracellular ROS by NAC significantly inhibited alantolactone-induced cell death (Fig. 4C). These results indicate that alantolactone may induce cell apoptosis by the generation of ROS in RKO cells.

Alantolactone induces apoptosis in the mitochondrial pathway. Mitochondria are a major source of ROS and excessive ROS accumulation leads to oxidative stress and ultimately apoptosis (27). To analyze whether mitochondria are involved in alantolactone-induced apoptosis, the changes of MMP using $\mathrm{DiOC}_{6}(3)$ staining were analyzed. Alantolactone resulted in marked depolarization in mitochondria (Fig. 5A). In addition, mitochondria usually undergo marked morphological changes
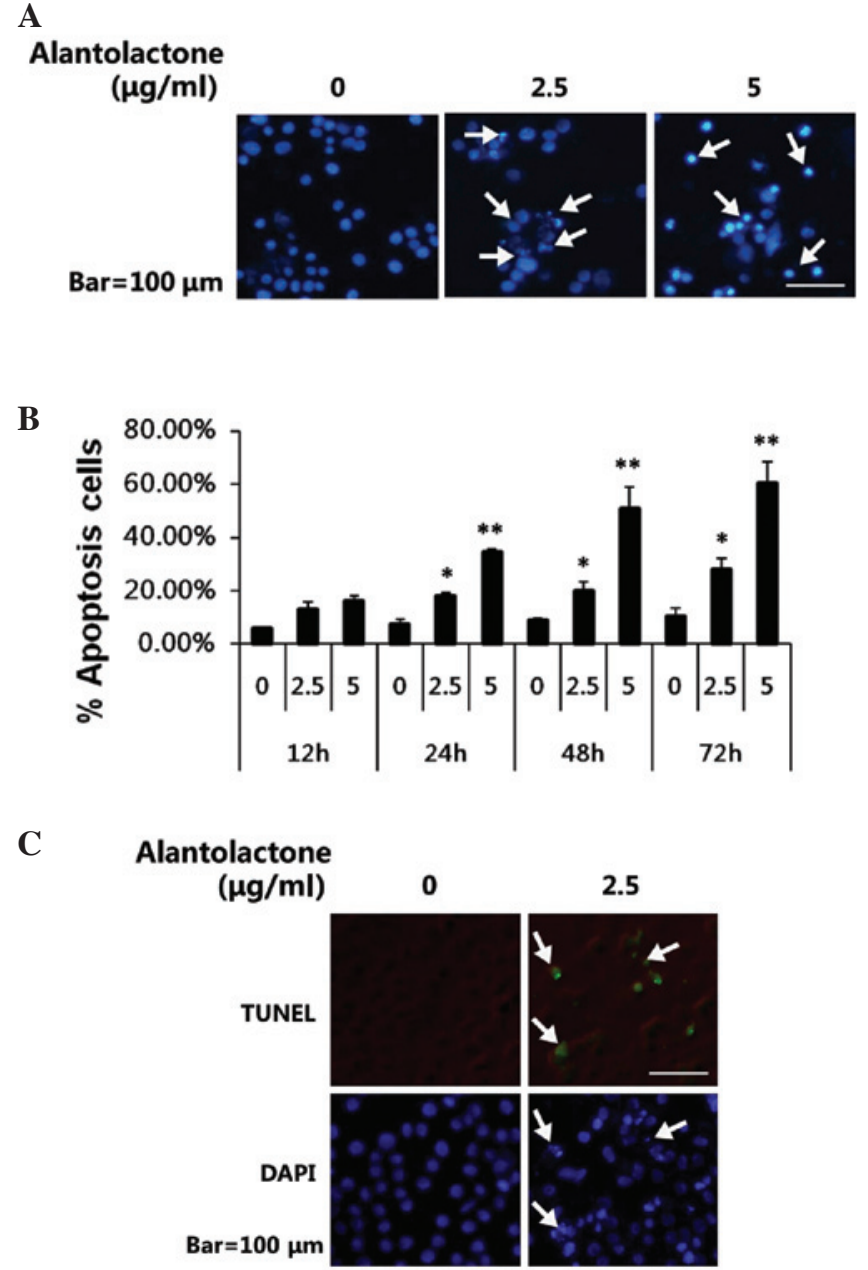

Figure 3. Effects of alantolactone on apoptosis in colon cancer cells. (A) Effect of alantolactone on the morphology of the nuclear chromatin in RKO cells. Cells were treated with $0,2.5$ and $5 \mu \mathrm{g} / \mathrm{ml}$ alantolactone for $24 \mathrm{~h}$, fixed and stained with DAPI. Arrows indicate apoptotic bodies in apoptotic cells. (B) RKO cells were treated with $0,2.5$ and $5 \mu \mathrm{g} / \mathrm{ml}$ alantolactone for 12 , 24,48 and $72 \mathrm{~h}$. Apoptotic cells were quantified by counting a minimum of four fields of 100 cells/field. (C) RKO cells were treated with 0 and $2.5 \mu \mathrm{g} / \mathrm{ml}$ alantolactone for $24 \mathrm{~h}$. Apoptotic cells were detected by TUNEL staining (green) and also confirmed by DAPI staining. Arrows show apoptotic bodies in apoptotic cells.

during the early stages of apoptosis (28). To visualize the effect of alantolactone on mitochondrial morphology, mitochondrially-targeted green fluorescent protein (mito-GFP) plasmids were transfected into RKO cells. As shown in Fig. 5B, mito-GFP expressing RKO cells revealed a markedly fragmented mitochondrial phenotype when treated with alantolactone, suggesting that depolarization in mitochondria and its fragmented phenotype are involved in alantolactone-induced apoptosis in RKO cells.

Caspase activation is generally considered to be a key hallmark of apoptosis (14). Mitochondria are involved in a variety of key events leading to apoptosis, including the release of caspase activators, the production of ROS and participation in regulation of proapoptotic and antiapoptotic Bcl-2 family proteins (15). A decrease in mitochondrial membrane potential disrupts the outer mitochondrial membrane, followed by the release of cytochrome $c$, activation of caspase-3, caspase- 9 and subsequent apoptosis. The effect of alantolactone on the 
A

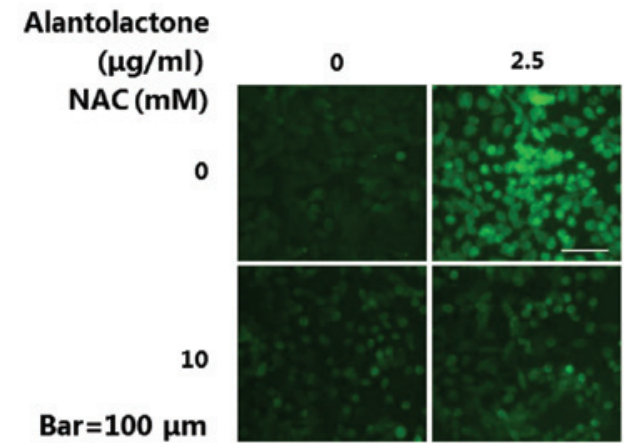

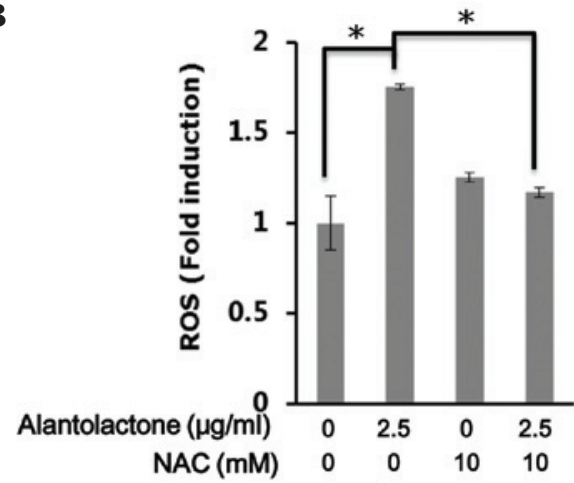

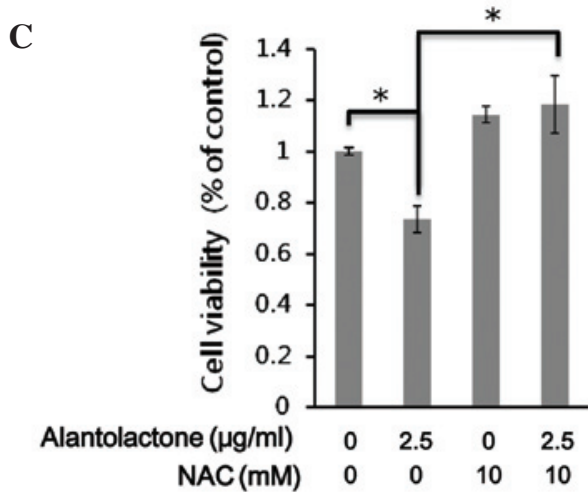

Figure 4. Alantolactone-generated ROS induces apoptosis in RKO cells. (A) RKO cells were pretreated with/without NAC (10 mM) $2 \mathrm{~h}$ prior to treatment with $2.5 \mu \mathrm{g} / \mathrm{ml}$ alantolactone. Following $24 \mathrm{~h}$ treatment, the cells were incubated with DCFH-DA (40 $\mu \mathrm{M}$ final) for 30 min and ROS production was detected by fluorescence microscopy. (B) Fluorescence intensity was measured with FLUOstar OPTIMA using $507 \mathrm{~nm}$ EX and $529 \mathrm{~nm}$ EM filter settings. (C) RKO cells were pretreated with/without NAC $(10 \mathrm{mM}) 2 \mathrm{~h}$ prior to treatment with $2.5 \mu \mathrm{g} / \mathrm{ml}$ alantolactone. Following treatment for $24 \mathrm{~h}$, cell viability was determined by MTT assay. NAC, N-acetylcysteine.

\section{A Alantolactone}

$(\mu \mathrm{g} / \mathrm{ml})$

$$
\mathrm{NAC}(\mathrm{mM})
$$

$\operatorname{Bar}=100 \mu \mathrm{m}$
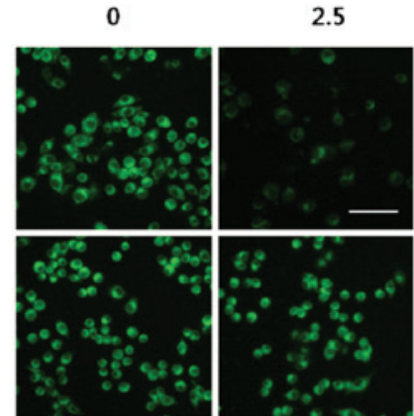

C
B

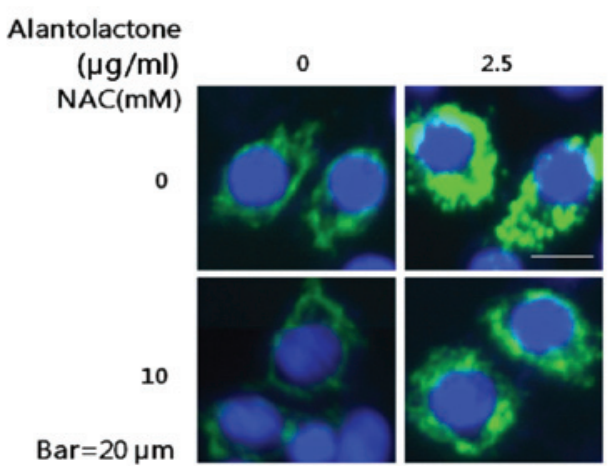

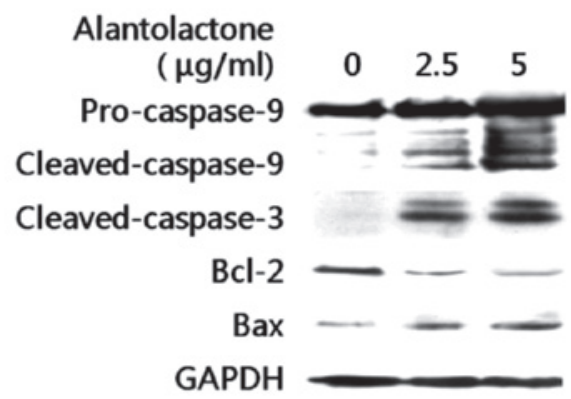

Figure 5. Effect of alantolactone on mitochondrial membrane potential, mitochondria injury and caspase activation. (A) RKO cells were treated with/without $\mathrm{NAC}(10 \mathrm{mM}) 2 \mathrm{~h}$ prior to treatment with $2.5 \mu \mathrm{g} / \mathrm{ml}$ alantolactone. Following $12 \mathrm{~h}$ alantolactone treatment, cells were incubated with DiOC 6 (3) for $30 \mathrm{~min}$, MMP levels were detected by fluorescence microscopy. (B) RKO cells were transfected with a mitochondrially targeted green fluorescent protein (mito-GFP) plasmid to visualize mitochondrial networks. Following $24 \mathrm{~h}$, cells were treated with/without NAC $(10 \mathrm{mM}) 2 \mathrm{~h}$ prior to treatment with $2.5 \mu \mathrm{g} / \mathrm{ml}$ alantolactone. Following $12 \mathrm{~h}$ treatment, the fragmented mitochondrial phenotype was detected by fluorescence microscopy. (C) RKO cells were incubated with $0,2.5$ and $5 \mu \mathrm{g} / \mathrm{ml}$ alantolactone for $24 \mathrm{~h}$. Cell lysates were prepared and subjected to SDS-PAGE. Pro-caspase-9, cleaved-caspase-9, cleaved-caspase-3, Bcl-2 and Bax were determined by western blotting using specific antibodies. NAC, N-acetylcysteine. 


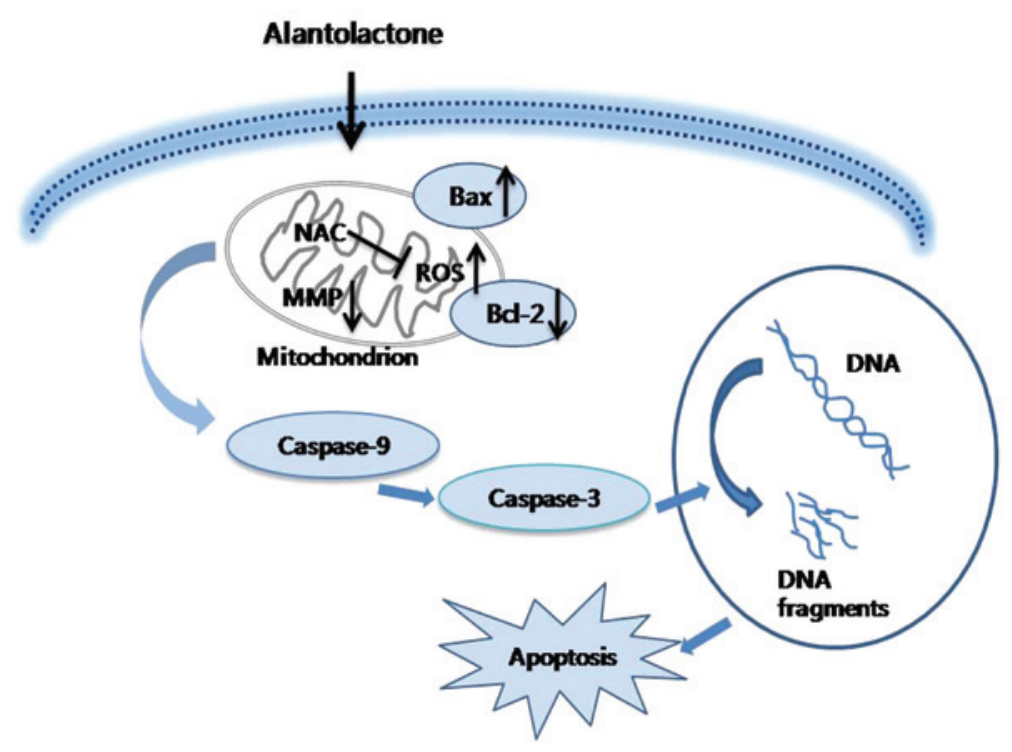

Figure 6. Proposed apoptotic signaling pathway activated by alantolactone in RKO cells.

activation of caspase- 3 and -9 , which are crucial initiators and effectors, respectively, was investigated. Alantolactone activated caspase- 3 and -9 , following decreased procaspase- 9 levels (Fig. 5C). To investigate the cell mechanism underlying alantolactone-induced apoptosis in RKO cells, Bcl-2 and Bax expression was analyzed. The expression levels of $\mathrm{Bcl}-2$ decreased and Bax expression increased in a dose-dependent manner (Fig. 5C). These results indicate that alantolactone induced RKO cell apoptosis-involved proteins from the $\mathrm{Bcl}-2$ family and caspase activation.

\section{Discussion}

Considerable attention has been focused on identifying a naturally occurring bioactive composition capable of inhibiting, retarding or reversing the carcinogenic process, particularly the development of colorectal cancer. In the colon, the elimination of transformed cells via apoptosis induction is considered to be a crucial step for the treatment of colorectal cancer (29).

In the present study, alantolactone was observed to be cytotoxic in vitro and to induce apoptosis in colorectal cancer RKO cells. To study the mechanism of alantolactone-induced apoptosis, the effects of alantolactone on ROS generation and MMP changes were investigated. The results showed that the accumulation of ROS was detected when treated with alantolactone (Fig. 4). To identify the role of ROS in alantolactone-induced apoptosis, NAC was used as a ROS scavenger, which may protect cells against oxidative damage by reacting with $\mathrm{H}_{2} \mathrm{O}_{2}$ as a direct antioxidant and increasing the cytoplasmic reserve of glutathione (30). If ROS production mediates alantolactone-induced cell death, we hypothesize that NAC is able to inhibit alantolactone-induced cell death. In the present study, NAC was observed to block intracellular ROS generation and further suppress alantolactone-induced apoptosis in RKO cells.

Mitochondria are a major source of ROS production and excessive ROS accumulation may lead to oxidative stress, induce decreased MMP and promote mitochondria-dependent apoptosis (27). Observations of the current study indicate that alantolactone causes marked depolarization in mitochondria. In addition, mitochondria undergo marked morphological changes during alantolactone-induced apoptosis. Thus, these results suggest that alantolactone-induced apoptosis is involved in ROS generation and mitochondrial dysfunction.

Mitochondria play vital roles in apoptosis induced by caspase-dependent and -independent pathways. Caspase- 3 is a key apoptotic executive caspase and is activated by proteolytic cleavage by caspase- 8 and -9 (31,32). In the current study, caspase-3 and -9 activation was observed to be involved in alantolactone-induced apoptosis, as indicated by decreased pro-caspase- 9 and increased cleavage of caspase-3.

The Bcl-2 protein family, whose members may be antiapoptotic or proapoptotic, regulates cell death by controlling mitochondrial membrane permeability during apoptosis $(33,34)$. Bcl-2 is a potent antiapoptotic factor, whereas $\mathrm{Bax}$, an antagonist of $\mathrm{Bcl}-2$, is inserted into the outer membrane of the mitochondria, allowing for the release of cytochrome $c$ and initiating apoptosis. It is therefore suggested that Bcl-2 family proteins may be involved in apoptosis induced by alantolactone. The present study showed that Bcl-2 decreased and Bax increased in alantolactone-induced apoptosis. These results indicate that treatment with alantolactone leads to a shift from an antiapoptotic to a proapoptotic state, resulting in the activation of capsase-3. Thus, alantolactone induces mitochondria-dependent apoptotic pathways in RKO cells, which involves the suppression of Bcl-2, elevation of Bax and activation of caspase- 9 and -3 .

In summary, the current study is the first to demonstrate that alantolactone inhibits cell proliferation and induces apoptosis via a ROS-mediated mitochondrial dependent pathway in RKO cells. Downregulation of Bcl-2, upregulation of Bax and activation of caspase- 3 and -9 are involved in this process (Fig. 6). Therefore, alantolactone is an attractive agent for human colon cancer research and may become a potent chemotherapeutic agent in colon cancer. 


\section{Acknowledgements}

This study was supported by grants from the Fundamental Research Funds for the Central Universities (no. 11QNJJ021), National Natural Science Foundation of China (nos. 30873409 , 30670220 and 31070318), the Fundamental Research Funds for the Central Universities (no. 11QNJJ021), Cultivation Fund of the Scientific and Technical Innovation Project of Northeast Normal University (no. NENU-STB07008), Administration of Traditional Chinese Medicine of Jilin Province (nos. 2010pt068 and 2011-zd17) and the Research Foundation of Jilin Provincial Science and Technology Development (nos. 20100911, 200805131 and 201201065).

\section{References}

1. Jemal A, Murray T, Ward E, et al: Cancer statistics, 2005. CA Cancer J Clin 55: 10-30, 2005.

2. Gustin DM and Brenner DE: Chemoprevention of colon cancer: current status and future prospects. Cancer Metastasis Rev 21: 323-348, 2002

3. Fauzi AN, Norazmi MN and Yaacob NS: Tualang honey induces apoptosis and disrupts the mitochondrial membrane potential of human breast and cervical cancer cell lines. Food Chem Toxicol 49: 871-878, 2011.

4. Hsu HF, Houng JY, Kuo CF, Tsao N and Wu YC: Glossogin, a novel phenylpropanoid from Glossogyne tenuifolia, induced apoptosis in A549 lung cancer cells. Food Chem Toxicol 46: 3785-3791, 2008.

5. Luo M, Liu X, Zu Y, et al: Cajanol, a novel anticancer agent from Pigeonpea [Cajanus cajan (L.) Millsp.] roots, induces apoptosis in human breast cancer cells through a ROS-mediated mitochondrial pathway. Chem Biol Interact 188: 151-160, 2010.

6. Cantrell CL, Abate L, Fronczek FR, et al: Antimycobacterial eudesmanolides from Inula helenium and Rudbeckia subtomentosa. Planta Med 65: 351-355, 1999.

7. Konishi T, Shimada Y, Nagao T, Okabe H and Konoshima T: Antiproliferative sesquiterpene lactones from the roots of Inula helenium. Biol Pharm Bull 25: 1370-1372, 2002.

8. Dirsch VM, Stuppner H and Vollmar AM: Cytotoxic sesquiterpene lactones mediate their death-inducing effect in leukemia T cells by triggering apoptosis. Planta Med 67: 557-559, 2001.

9. Lawrence NJ, McGown AT, Nduka J, Hadfield JA and Pritchard RG: Cytotoxic Michael-type amine adducts of alpha-methylene lactones alantolactone and isoalantolactone. Bioorg Med Chem Lett 11: 429-431, 2001.

10. Shi Y, Bao YL, Wu Y, et al: Alantolactone inhibits cell proliferation by interrupting the interaction between Cripto-1 and activin receptor type II A in activin signaling pathway. J Biomol Screen 16: 525-535, 2011.

11. Bellamy CO, Malcomson RD, Harrison DJ and Wyllie AH: Cell death in health and disease: the biology and regulation of apoptosis. Semin Cancer Biol 6: 3-16, 1995.

12. Reed JC: Apoptosis-regulating proteins as targets for drug discovery. Trends Mol Med 7: 314-319, 2001.

13. Kaufmann SH, Kottke TJ, Martins LM, Henzing AJ and Earnshaw WC: Analysis of caspase activation during apoptosis. Curr Protoc Cell Biol Chapter 18: Unit 18.2, 2001.

14. Danial NN and Korsmeyer SJ: Cell death: critical control points. Cell 116: 205-219, 2004.

15. Murphy KM, Ranganathan V, Farnsworth ML, Kavallaris M and Lock RB: Bcl-2 inhibits Bax translocation from cytosol to mitochondria during drug-induced apoptosis of human tumor cells. Cell Death Differ 7: 102-111, 2000.
16. Chatterjee S, Kundu S and Bhattacharyya A: Mechanism of cadmium induced apoptosis in the immunocyte. Toxicol Lett 177: 83-89, 2008.

17. Pathak N and Khandelwal S: Role of oxidative stress and apoptosis in cadmium induced thymic atrophy and splenomegaly in mice. Toxicol Lett 169: 95-108, 2007.

18. Martindale JL and Holbrook NJ: Cellular response to oxidative stress: signaling for suicide and survival. J Cell Physiol 192: 1-15, 2002.

19. Zhou YJ, Zhang SP, Liu CW and Cai YQ: The protection of selenium on ROS mediated-apoptosis by mitochondria dysfunction in cadmium-induced LLC-PK(1) cells. Toxicol In Vitro 23: 288-294, 2009.

20. Yang JS, Chen GW, Hsia TC, et al: Diallyl disulfide induces apoptosis in human colon cancer cell line (COLO 205) through the induction of reactive oxygen species, endoplasmic reticulum stress, caspases casade and mitochondrial-dependent pathways. Food Chem Toxicol 47: 171-179, 2009.

21. Wei A, Zhou D, Xiong C, Cai Y and Ruan J: A novel non-aromatic B-ring flavonoid: isolation, structure elucidation and its induction of apoptosis in human colon HT-29 tumor cell via the reactive oxygen species-mitochondrial dysfunction and MAPK activation. Food Chem Toxicol 49: 2445-2452, 2011.

22. Wang L, Xu ML, Hu JH, Rasmussen SK and Wang MH: Codonopsis lanceolata extract induces $\mathrm{G} 0 / \mathrm{G} 1$ arrest and apoptosis in human colon tumor HT-29 cells - involvement of ROS generation and polyamine depletion. Food Chem Toxicol 49: 149-154, 2011.

23. Zhou L, Bao YL, Zhang Y, et al: Knockdown of TSP50 inhibits cell proliferation and induces apoptosis in P19 cells. IUBMB Life 62: 825-832, 2010.

24. Zhang Y, Zhou L, Bao YL, et al: Butyrate induces cell apoptosis through activation of JNK MAP kinase pathway in human colon cancer RKO cells. Chem Biol Interact 185: 174-181, 2010.

25. Rizzuto R, Nakase H, Darras B, et al: A gene specifying subunit VIII of human cytochrome c oxidase is localized to chromosome 11 and is expressed in both muscle and non-muscle tissues. J Biol Chem 264: 10595-10600, 1989.

26. Zafarullah M, Li WQ, Sylvester J and Ahmad M: Molecular mechanisms of $\mathrm{N}$-acetylcysteine actions. Cell Mol Life Sci 60: 6-20, 2003

27. Rego AC and Oliveira CR: Mitochondrial dysfunction and reactive oxygen species in excitotoxicity and apoptosis: implications for the pathogenesis of neurodegenerative diseases. Neurochem Res 28: 1563-1574, 2003.

28. Shen Z, Shen J, Chen M, Li Q and Hong C: Morphological changes of mitochondria in apoptosis of esophageal carcinoma cells induced by $\mathrm{As}(2) \mathrm{O}(3)$. Zhonghua Bing Li Xue Za Zhi 29: 200-203, 2000 (In Chinese).

29. Gryfe R, Swallow C, Bapat B, et al: Molecular biology of colorectal cancer. Curr Probl Cancer 21: 233-300, 1997.

30. Sadowska AM, Manuel Y-Keenoy B and De Backer WA: Antioxidant and anti-inflammatory efficacy of NAC in the treatment of COPD: discordant in vitro and in vivo dose-effects: a review. Pulm Pharmacol Ther 20: 9-22, 2007.

31. Budihardjo I, Oliver H,Lutter M, Luo X and Wang X: Biochemical pathways of caspase activation during apoptosis. Annu Rev Cell Dev Biol 15: 269-290, 1999.

32. Kroemer G and Martin SJ: Caspase-independent cell death. Nat Med 11: 725-730, 2005.

33. Adams JM and Cory S: The Bcl-2 protein family: arbiters of cell survival. Science 281: 1322-1326, 1998.

34. Yin XM: Signal transduction mediated by Bid, a pro-death Bcl-2 family proteins, connects the death receptor and mitochondria apoptosis pathways. Cell Res 10: 161-167, 2000.

35. Nomura M, Shimizu S, Ito T, et al: Apoptotic cytosol facilitates Bax translocation to mitochondria that involves cytosolic factor regulated by Bcl-2. Cancer Res 59: 5542-5548, 1999. 